\title{
«Non, monsieur Adenauer, chez nous, personne ne songe à construire de nouvelles églises »
}

La politique culturelle des autorités et les demandes spirituelles des premiers Kaliningradois.

\section{Evgueni Maslov}

Traducteur : Alexandre Smirnov

\section{OpenEdition}

\section{Journals}

Édition électronique

URL : https://journals.openedition.org/rbnu/2818

DOI : $10.4000 /$ rbnu. 2818

ISSN : 2679-6104

\section{Éditeur}

Bibliothèque nationale et universitaire de Strasbourg

\section{Édition imprimée}

Date de publication : 1 mai 2012

Pagination : 26-38

ISSN : 2109-2761

\section{Référence électronique}

Evgueni Maslov, « « Non, monsieur Adenauer, chez nous, personne ne songe à construire de nouvelles églises » », La Revue de la BNU [En ligne], 5 | 2012, mis en ligne le 01 mai 2012, consulté le 25 août 2021. URL : http://journals.openedition.org/rbnu/2818 ; DOI : https://doi.org/10.4000/rbnu.2818

Ce document a été généré automatiquement le 25 août 2021.

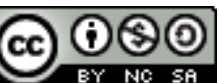

La Revue de la BNU est mise à disposition selon les termes de la Licence Creative Commons Attribution - Pas d'Utilisation Commerciale - Partage dans les Mêmes Conditions 4.0 International. 


\section{«Non, monsieur Adenauer, chez nous, personne ne songe à construire de nouvelles églises »}

La politique culturelle des autorités et les demandes spirituelles des premiers Kaliningradois ${ }^{1}$.

\section{Evgueni Maslov}

Traduction : Alexandre Smirnov

1 En 1960, à la session du Soviet suprême de la RSFSR ${ }^{2}$, le président du Comité exécutif régional de Kaliningrad, Z. F. Slaykovsky, prononçait un discours ardent, dans lequel il faisait le bilan des quinze ans de la politique des autorités locales dans le domaine de la culture et de la spiritualité. Voici les fragments les plus significatifs de cette intervention :

Lors d'un rassemblement récent des hitlériens d'aujourd'hui à Düsseldorf, Adenauer a déclaré, en attisant les velléités revanchardes, que Königsberg et ses terres adjacentes étaient un désert, une zone morte, où il n'y avait pas d'églises ni une quelconque vie culturelle et spirituelle. Que peut-on dire au sujet de ces paroles du vieil intrigant bonnois? Oui, il y a quinze ans, Königsberg et ses terres adjacentes représentaient en effet un désert, une zone morte. Les hitlériens ont laissé derrière eux une économie complètement détruite, ils avaient utilisé des tonnes d'explosifs, des centaines de milliers d'engins divers et de mines. Tout cela se faisait conformément à la tactique de la "terre brûlée". Des ruines, des amas de briques et de gravillons, des champs défigurés par les tranchées et couverts de mauvaises herbes - voici ce que la région de Kaliningrad formée en 1946 représentait. Mais en répondant à l'appel du parti et du gouvernement, des centaines de milliers de travailleurs de notre pays sont venus s'installer dans cette nouvelle région soviétique... Les hommes soviétiques ont apporté avec eux un nouveau mode de vie et de nouvelles requêtes spirituelles. Non, monsieur Adenauer, chez nous personne ne songe à construire de nouvelles églises. Elles ne sont pas nécessaires, tout simplement. Dans notre région vivent des gens cultivés et areligieux dans leur conception du monde. Cependant les anciennes églises allemandes sont restées. Nous ne les détruisons pas. Certaines d'entre elles, comme la cathédrale, sont même sous la protection de l'État. D'autres, ayant une valeur architecturale, servent aux 
Kaliningradois en tant que cinémas et musées. La vie spirituelle des hommes soviétiques est riche et variée. Aujourd'hui les habitants de notre région ont plus de cent mille appareils de radio, plus de cent mille spectateurs regardent chaque jour les émissions télévisées de Kaliningrad...

Ensuite Slaykovsky évoque d'autres preuves statistiques de la richesse de la vie culturelle et spirituelle de la région - une société philharmonique régionale, 400 maisons de la culture, salles de concerts et clubs, 28 universités populaires, 282 bibliothèques, 900 cercles artistiques d'amateurs, 900 concerts donnés directement dans les champs par des groupes de propagande...

2 La position du haut fonctionnaire de Kaliningrad est tout à fait claire. Dans l'article présent, nous tenterons de comprendre dans quelle mesure elle correspondait aux requêtes spirituelles réelles des habitants de la région.

\section{Les ruines de Königsberg en 1945}

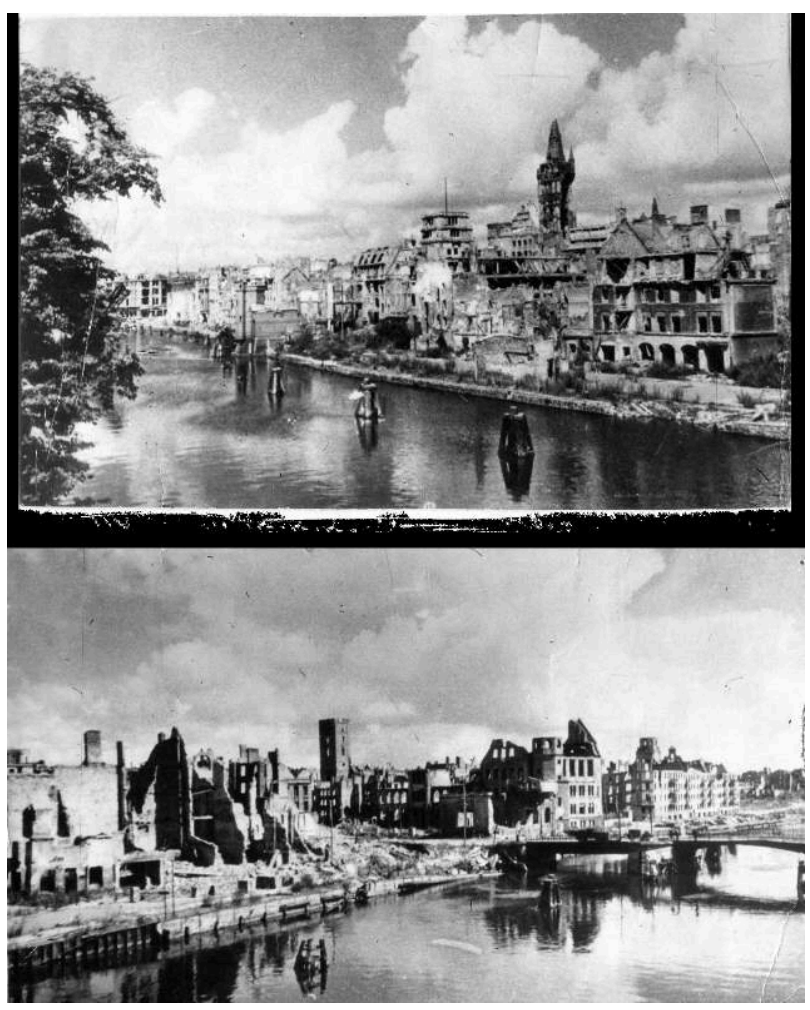

En 1945 Willi Schedler, dernier architecte de la ville, s'est mis à la disposition des pouvoirs soviétiques pour les aider à dresser des cartes et à reconstituer les plans de celle-ci. Accompagné par des architectes et des militaires soviétiques, Schedler a pris plusieurs photos de la ville en ruines. Mort en 1947, il a laissé une centaine de clichés qui appartiennent actuellement à Avenir Ovsyanov, spécialiste et expert auprès du Département de la protection du patrimoine de la région de Kaliningrad.

Aujourd'hui, ces photographies sont considérées comme une part importante du patrimoine de la ville. Photographie Willi Schedler

\section{Les colons et leurs besoins spirituels}

3 Un des processus les plus remarquables du développement social et démographique de la région de Kaliningrad à l'époque d'après-guerre fut l'obratnitchestvo. Cette notion désignait le retour des migrants volontaires dans leur région natale, phénomène alors largement répandu : rien qu'en 1948-1953, 260174 migrants sur 510063 avaient quitté 
la région de Kaliningrad. Au cours de l'année 1956, 61600 personnes sont venues dans la région et 53900 personnes en sont parties. L'obratnitchestvo est devenu un phénomène national. Sur l'île de Sakhaline, sur 135000 personnes arrivées en 1950, 74000 sont retournées chez elles, et parmi les 130700 colons de 1953, il y avait 115300 obratniks.

4 Parmi les nombreuses causes de l'obratnitchestvo, les plus décisives étaient d'ordre matériel, financier, psychologique et spirituel. Nous les examinerons tour à tour. L'historiographie régionale nous montre que les considérations utilitaires constituaient le motif principal de migration dans une région - désir d'échapper à la famine et à la misère des lieux où l'on vivait, ou de recevoir des avantages. Les gens, parce qu'ils voulaient y croire, écoutaient les recruteurs qui vantaient les grandes possibilités s'ouvrant aux personnes qui accepteraient de partir pour l'ancienne Prusse-Orientale. Mais la réalité ne justifia pas les attentes.

5 Dans les premières années de l'après-guerre, Kaliningrad était pratiquement en ruines. En 1954, un journaliste local écrivait : « Nous nous rappelons le Königsberg des années 1945-1946. Le château royal et l'université détruits par les incursions de l'aviation anglaise, les murs sombres qui restaient de la cathédrale brûlée, les ruines des bâtiments dans les rues, les tas de gravats sur les chaussées, les feux ternes des lampes à pétrole dans les maisons épargnées, les voies de tramway couvertes de débris, les conduites d'eau rouillées, à sec; le sifflement du vent dans les ateliers détruits des usines, l'abandon dans le port, les enseignes arrachées des magasins ». L'architecte en chef de la ville de 1948 à 1958, D. Navalikhine, avançait les chiffres suivants : l'ensemble des habitations de Königsberg avant la guerre faisait près de $6000000 \mathrm{~m}^{3}$, or en octobre 1948 il ne restait que $1035000 \mathrm{~m}^{3}$ (soit 17,25\%); sur 13368 bâtiments, 8355 étaient détruits, soit $62,5 \%$.

6 Aux problèmes d'ordre matériel dont souffraient les colons de Kaliningrad s'ajoutaient des difficultés d'ordre spirituel et psychologique. Il a fallu quelques décennies après 1945 avant que n'apparaissent des générations de Kaliningradois pour qui cette nouvelle terre russe était devenue familière - et qui étaient devenus indifférents à son passé prusso-germanique ou aux images significatives d'une autre culture, images qui étaient toujours présentes dans l'architecture et la sculpture locales, dans la toponymie ou dans les inscriptions diverses.

7 Le temps a rapproché les gens et la terre au fur et à mesure. De nouvelles générations de Kaliningradois apparurent, les cimetières russes s'élargirent et devinrent une racine nationale très solide - les tombes des pères attachent les gens de façon plus certaine que n'importe quelle ancre... Pour des raisons naturelles, les premiers colons regardaient l'ancienne Prusse-Orientale de façon très différente. Le fait de l'annexion juridique à la république russe d'un petit coin sur la côte de la mer Baltique ne les empêchait pas de le percevoir comme une terre étrangère. L'antithèse "indigène étranger " s'aggrave invariablement dans la conscience collective d'un groupe social, quand il se trouve dans un état marginal. Les colons de Kaliningrad étaient des marginaux typiques, et cela à différents niveaux - chronologique, spatial et culturel. C'étaient des gens déjà sortis de la guerre, mais pas encore adaptés au temps de paix ; des gens qui avaient déjà quitté leur région d'origine, mais qui ne s'étaient pas encore habitués à la nouvelle; des gens plongés dans un espace spirituel qui leur était étranger, se trouvant dans une zone géopolitique frontalière et n'ayant pas sur cette 
terre les points d'ancrage d'un natif. A. I. Ryjova, jeune fille venue dans la région à dixsept ans, se rappelle :

Il fallait naître et grandir ici. C'était une autre psychologie, une autre conception de l'éternité. En premier lieu, cela se sentait en regardant les espaces sacrés - les églises luthériennes et catholiques. Nos églises et nos temples sont plus marqués par la bonté en quelque sorte, plus accueillants. L'austérité et l'architecture anguleuse des cathédrales locales ne correspondent pas à notre caractère russe. Je pouvais les admirer en tant qu'œuvres architecturales, mais je ne les voyais pas comme un espace où l'on pouvait me comprendre et me réconforter. Leur aspect extérieur suggérait l'inverse. Il en émanait quelque souffle froid et étranger. Les jours de pluie, la ville produisait une impression mélancolique, opprimante par l'étroitesse des rues, l'austérité des bâtiments. On avait alors une sensation du caractère provisoire de notre séjour et on sentait en particulier à quel point nous étions des étrangers ici.

\section{La cathédrale en 1975}

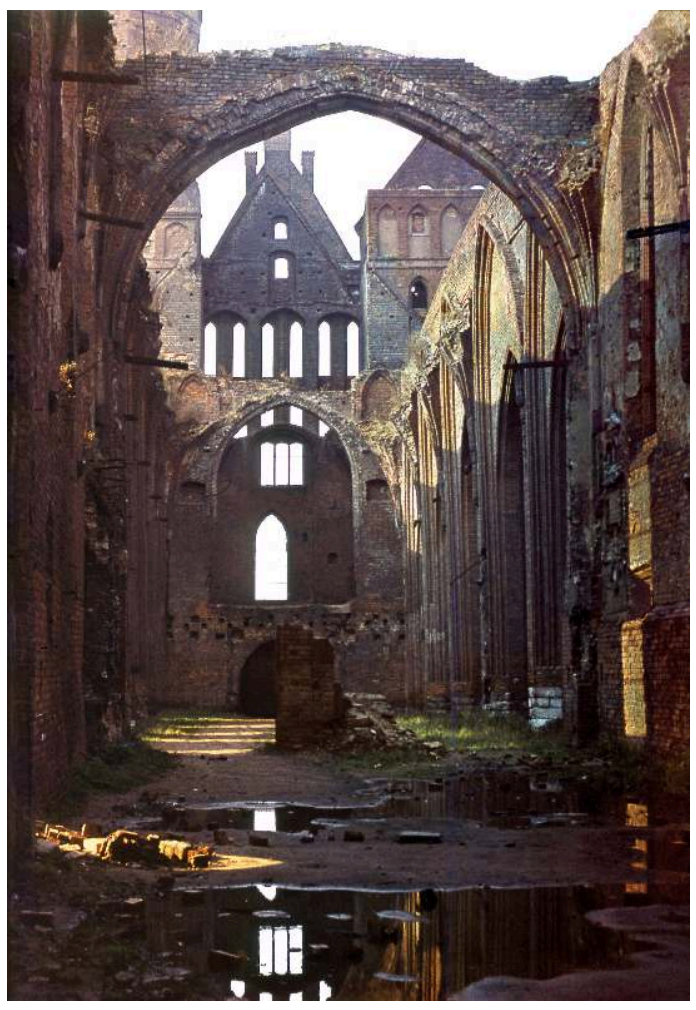

Photographie Yuri Bardun

En écho à ces paroles, l'historienne I. Belintseva apporte un commentaire culturel très précieux :

Au cours de la mise en valeur de l'héritage inconnu de Königsberg, le rôle essentiel fut joué par l'environnement architectural, l'ensemble des éléments du paysage municipal restés intacts après les bombardements et les tirs d'artillerie. Pour les nouveaux habitants de la ville qui étaient venus du fin fond de l'URSS, le milieu urbain de Königsberg-Kaliningrad portait l'empreinte d'une culture spirituelle et matérielle hautement développée, mais d'un caractère essentiellement étranger, et de plus appartenant à leur pire ennemi, même s'il était vaincu. Les colons étaient pour l'essentiel originaires des petites villes et des villages de la Russie centrale et appartenaient, en vertu de leur origine, à la culture populaire avec des orientations 
et des valeurs spirituelles et matérielles tout à fait éloignées de leur nouvel environnement. optimistes. Comme la kolkhozienne A. F. Savitskaya, qui en 1948 rassurait les habitants de son village: «Allons, ne baissez pas les bras... Tout n'est jamais donné d'emblée, nous nous y habituerons. Un Russe est chez lui là où il vit paisiblement et en bonne harmonie». Mais un sentiment négatif envers cette nouvelle terre russe restait prédominant. Sa perception en tant que terre étrangère se renforçait par d'autres faits : par la présence des Allemands au début et la mémoire des crimes du nazisme, par la peur de la guerre qui semblait imminente partout (non seulement en URSS, mais aussi aux États-Unis ou en Europe occidentale), et particulièrement dans la région de Kaliningrad et sur l'île de Sakhaline. En 1955, il y eut quelques cas de communistes exclus du parti sous un prétexte significatif dans ce contexte : « pour cause de violation de la discipline du parti consistant dans le départ non autorisé de la région en raison de l'esprit de panique, provoqué par la crainte des complications internationales ». La situation d'enclave de la région et la police des frontières qui la surveillait ont joué leur rôle dans cette situation.

La perception de l'ancienne Prusse-Orientale comme une terre étrangère se manifestait en premier lieu chez les colons qui avaient gardé leur conscience culturelle nationale, et particulièrement chez les croyants orthodoxes qui se sont retrouvé privés de leurs lieux de culte et donc de la possibilité de s'appuyer sur la tradition orthodoxe dans leur nouvelle patrie. Ici, il faut donner une précision. Dans la région de Kaliningrad jusqu'en 1945, l'Église orthodoxe constituait une confession insignifiante [...] Les faits témoignant de l'existence, dans l'histoire d'avant-guerre de la nouvelle région russe, de communautés orthodoxes, d'églises, monastères et autres lieux sacrés orthodoxes étaient inconnus non seulement des premiers colons, mais aussi, semble-t-il, de la plupart des Kaliningradois contemporains. D'un point de vue religieux, l'ancienne Prusse-Orientale se présentait aux Russes arrivant ici comme une terre non orthodoxe, mais catholique et protestante.

11 On peut affirmer sans exagération qu'une partie des obratniks ont quitté la région justement pour des motifs religieux. On ignore dans quelles proportions. De même, on ne pourra jamais savoir au juste quel nombre d'obratniks ont quitté la région pour des raisons matérielles et financières. Aucune source ne donne ces chiffres, bien que dans les documents des organisations de migration on trouve des statistiques concernant les raisons des retours ${ }^{3}$.

12 Les colons qui décidaient de rester quand même aspiraient à aménager cette jeune région russe, à l'adapter à leurs traditions, leurs us et leurs coutumes. Dans la vie courante, on avait pleine conscience de la tâche socioculturelle qu'était la « russification » de la région. A. I. Ryjova, déjà mentionnée, disait à ce propos :

Je n'ai jamais vu une ville aussi verdoyante. À l'époque j'ai pensé que c'était un symbole. La jeune verdure - c'est la renaissance. J'avais le sentiment que je devais faire quelque chose pour cette ville, qui était sans doute belle. Quelque chose pour l'harmonie. Oui, j'étais alors trop jeune et pensais qu'il fallait faire de cette terre une terre russe. En somme, nous le pensions tous à l'époque.

$13 \mathrm{Au}$ titre de soutien moral, les croyants orthodoxes de la région de Kaliningrad souhaitaient avoir leurs églises. Certains extraits de lettres du colon A. I. Orechnikov au 
patriarche Alexis $1^{\text {er }}$ et au Conseil des affaires de l'Église orthodoxe russe en 1948 en témoignent très clairement :

Étant venu de Saratov pour m'installer chez ma fille, j'ai décidé d'aider le peuple russe d'une façon ou d'une autre [...] Il n'y a aucune église orthodoxe dans toute la région sur un espace qui va jusqu'à la Lituanie, mais un grand nombre d'églises catholiques et protestantes... Ce fait présente de grandes difficultés pour les Russes orthodoxes qui vivent ici et se sont installés dans cette contrée, pour nous, les patriotes ardents de notre patrie russe bien aimée... Ici se sont installées entre autres les familles des défenseurs de la patrie tombés pendant la guerre, qui désirent tant avoir un temple orthodoxe... Et ce petit temple russe orthodoxe, cette petite parcelle bien à nous, sera ici un rempart d'orthodoxie russe [...] Nous demandons à Votre Sainteté de soutenir notre requête [...] sur l'autorisation de l'ouverture ici de ce petit temple pour y élever nos prières vers Dieu, pour vous, pour votre santé et prospérité, pour notre Grand Dirigeant sage et les autorités, pour la puissante et glorieuse armée russe et pour tout le peuple orthodoxe russe. Car la Sainte Église orthodoxe n'a pas seulement la vertu des propriétés morales, elle est aussi la source de la force spirituelle du peuple orthodoxe russe, la source de son esprit puissant, l'esprit du patriotisme et de l'amour de la patrie, qui fait que notre puissant pays russe triomphe toujours de tous les désastres de la guerre s'abattant sur lui.

\section{Le caractère étranger de l'héritage spirituel : les tentatives de résolution politique du problème}

Les difficultés concernant l'adaptation socioculturelle et psychologique des premiers colons, comme le phénomène d'obratnitchestvo, ne pouvaient pas ne pas attirer l'attention des dirigeants soviétiques. Les autorités tentèrent de résoudre ces problèmes. On mit en place une résistance administrative et juridique à la « désertion de la région » et on prit des mesures pour l'amélioration des conditions de vie des colons. Des poursuites furent entamées contre les personnes parties de la région sans autorisation, afin de recouvrer les ressources dépensées par l'État pour leur émigration. On punissait les dirigeants qui ne prenaient pas les mesures nécessaires pour l'installation des kolkhoziens et des ouvriers. On organisait des conférences spéciales sur les questions de la qualité de l'accueil des colons, et il y avait de nombreuses commissions où l'on prenait des décisions parfois sévères. On attachait une grande importance à la propagande concernant la conscience socialiste et l'attitude stoïque à adopter envers toutes les difficultés « temporaires » de l'époque de l'après-guerre.

L'administration de la région comprenait aussi le problème du caractère étranger de l'héritage spirituel. Le secrétaire du Comité régional de la propagande I. P. Trifonov disait lors de l'une des premières réunions des militants régionaux du parti (le 27 septembre 1947) :

Notre région est formée par les terres de l'ancien État prussien, qui fut pendant longtemps le foyer de la réaction la plus noire, la place d'armes des incursions criminelles des impérialistes germaniques [...] un endroit où chaque pierre, chaque ferme nous était hostile, où chaque ville, chaque village, chaque maison gardaient les traces de l'individualisme bestial allemand [...] Sa population comprend des colons qui sont arrivés ici, provenant de tout le pays soviétique, des gens d'un niveau culturel et politique inégal, avec des habitudes, des coutumes et des traditions différentes. Pour unir ces gens [...] un travail d'éducation quotidien et minutieux est nécessaire. 
Six ans après, le premier secrétaire du Comité régional de Kaliningrad, V.E.Tchernychev, et le président du Comité exécutif régional de Kaliningrad, Z. F. Slaykovsky, écrivaient à N.S. Khrouchtchev à propos de l'importance du travail socioculturel pour un enracinement plus solide des colons dans la région. Somme toute, on avait compris le problème. Mais que faisait-on concrètement?

En premier lieu, on s'affairait à trois tâches, visant à éliminer le passé et à construire l'avenir : l'action culturelle, la création d'une histoire nouvelle de la région et la lutte contre l'héritage de la culture germanique. Le programme de l'action culturelle était typique de l'époque soviétique et supposait la création et l'organisation d'institutions scientifiques, scolaires et sanitaires, de théâtres, salles de concert, musées, maisons de la culture, parcs de culture et de repos, salles de conférence, cinémas, bibliothèques et réseaux de librairies, sans compter la radio, la presse, les organisations de la culture physique et du sport, les stades, les salles de sport... La réalisation de ce programme était complexe, mais on réussit à atteindre les objectifs chiffrés nécessaires. Et c'était l'essentiel, comme partout dans le pays des Soviets. Par exemple, l'usage même de la radio était plus important que le contenu de son programme. La masse des publications était plus importante que l'attention au lecteur [...] Les rapports sur les questions culturelles de la décennie d'après-guerre rappellent les rapports sur les rythmes des travaux sur les grands chantiers, et sont peu distincts, même structurellement, de ceux concernant le développement socioéconomique de la région. Les mots clés étaient « action" et «construction». Dans la région de Kaliningrad, on donnait aux chiffres concernant les actions culturelles nouvellement créées une valeur singulière également parce qu'il ne s'agissait pas d'une restauration, mais d'une création ex nihilo. La qualité et les composantes de l'activité des établissements culturels étaient presque les mêmes que partout dans l'Union soviétique : les mêmes repères socialistes, le même contrôle idéologique par le parti, le même formalisme. Les quelques différences étaient déterminées par la spécificité de l'héritage historique et culturel.

La région de Kaliningrad était privée de sa propre histoire. On l'a créée et implantée très efficacement dans la conscience des colons. Le passé du territoire de la région se divisait en trois périodes inégales: prussienne, germanique et soviétique. Chaque période était traitée sur la base du principe connu de l'historien russe M. N. Pokrovsky selon lequel l'histoire à l'époque soviétique n'était qu'une vision du passé formatée par la politique du moment. À l'époque où dans les conférences publiques données à Moscou, certains auteurs n'offensaient pas trop l'objectivité historique et classaient les Prussiens dans les tribus lituaniennes (parler de Baltes occidentaux aurait quand même été un choix plus juste), les propagandistes de la région de Kaliningrad disaient unanimement que les Prussiens étaient des Slaves. Cette idée révisionniste est parfaitement résumée dans le titre d'une émission de radio : "L'ancienne terre slave est récupérée par ses propriétaires légaux ». La volonté de donner aux colons des points d'appui nationaux allait parfois jusqu'à l'absurde. A la question « pourquoi peuton appeler Kaliningrad ville natale ", le député du Conseil de la ville Kharkov répondit : "c'est parce qu'elle se trouve sur une terre slave et dans l'Antiquité c'était une vraie ville slave ». En réalité, Königsberg n'a jamais été une ville slave, ni même une ville prussienne, sauf si l'on estime que son origine était l'ancien village de Tvangste. Lors des conférences internationales issues de la Seconde Guerre mondiale, Staline luimême appelait le territoire de l'ancienne Prusse-Orientale « une terre slave ». Son point de vue était notamment reflété dans l'article concernant la région de Kaliningrad de la 
Grande encyclopédie soviétique. Il faut dire que cette conception de Staline n'était pas seulement définie par la nécessité politique. Elle avait une base historiographique, mais celle-ci était surannée. Au milieu du $18^{\mathrm{e}}$ siècle, M. V. Lomonosov, conformément aux idées courantes de son temps, classait les Prussiens dans les tribus slaves occidentales. Or, selon les observations des archéologues modernes, les Baltes occidentaux ne sont certes pas des Slaves, mais leur origine ethnique est étroitement liée à la formation de certaines unions de tribus slaves, en particulier les Mazoviens et les Krivitches. Et si l'on prend en considération le fait que les peuples baltes (les Lituaniens et les Lettons) faisaient partie de la communauté nationale soviétique, alors la présupposition idéologique sur les racines slaves de la terre de Kaliningrad n'était pas du tout antiscientifique. De plus, les propagandistes soviétiques auraient pu utiliser l'héritage anti-nordique de Lomonosov plus efficacement, s'ils avaient prétendu que le nom de leur patrie et du peuple venait des bords de la mer Baltique. Jusqu'à présent l'une des hypothèses de travail sur l'origine de l'appellation des Prussiens est la conception de T. Narbout (datant du début du $19^{\mathrm{e}}$ siècle) selon laquelle leur nom d'origine est dérivé de l'affluent du Niémen, la Ross. De là il ne restait qu'un pas à faire vers une assertion encore plus radicale: les Prussiens étant slaves, le prince prussien Riourik qui est le fondateur reconnu du premier État des Slaves orientaux - la Russie kiévienne - est donc russe. Sa terre natale, c'est-à-dire la région de Kaliningrad, devient ainsi le berceau de l'État russe. Mais il restait un problème : pour les historiens soviétiques de cette époque, Riourik était une invention des annalistes. Après la mort de Staline, les déclarations sur le passé slave de la région ont rapidement disparu de la rhétorique publique. Et dans le nouveau volume de la Grande encyclopédie soviétique, ainsi que dans les autres éditions, on a « rendu » aux Prussiens leur langue maternelle et leur culture. 
Königsberg à Kaliningrad : arrêt de bus sur la place de la Victoire ; en arrière-plan, la nouvelle église orthodoxe

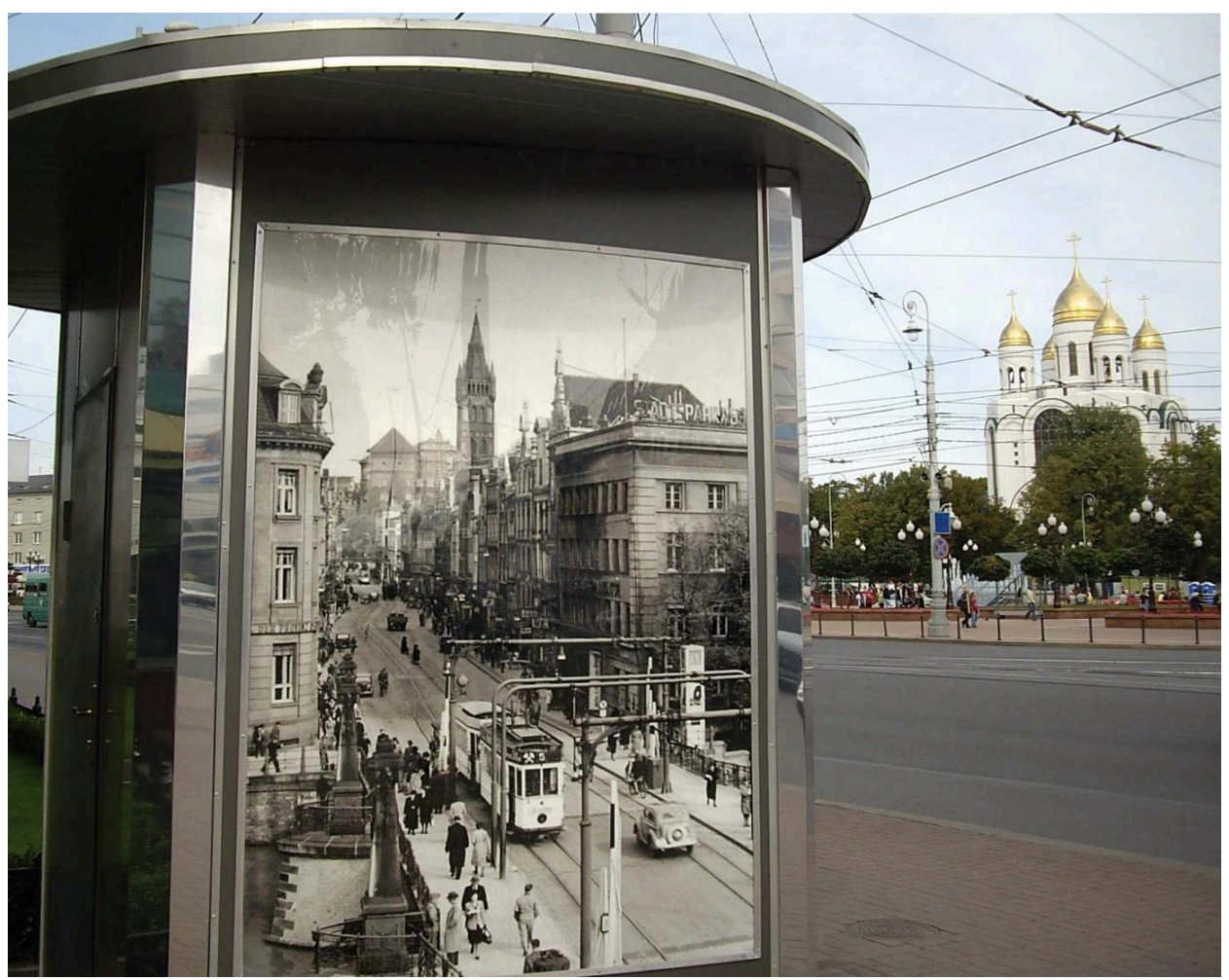

Photographie Yuri Bardun

La période germanique du passé de la région de Kaliningrad (dès le $13^{\mathrm{e}}$ siècle) était en général dépeinte par les propagandistes sous des couleurs sombres. On ne négligeait aucun détail, comme le fait qu'un des fondateurs de la théorie sur l'origine nordique de la Russie, « le falsificateur connu de l'histoire nationale » G. S. Bayer, était originaire de Königsberg et professeur à l'université de cette ville. On ne décrivait sous un jour favorable que des épisodes choisis de la présence de l'armée russe sur le territoire de la Prusse-Orientale - la lutte contre l'ordre teutonique, la guerre de Sept Ans, les campagnes contre Napoléon. En revanche, on préférait ne pas mentionner les événements de la Première Guerre mondiale, et surtout ceux de la guerre civile, ainsi que les émigrés russes de Königsberg.

20 La vie des colons n'était certainement pas facilitée par l'idée qu'auparavant, en cet endroit, " chaque pierre, chaque ferme [leur] était hostile », mais la troisième période de l'histoire de la région devait être perçue de façon d'autant plus radieuse. Cette période commençait par l'entrée de l'armée rouge sur les terres prussiennes et était présentée sous un jour héroïque, celui du patriotisme et des grands espoirs d'un futur heureux pour cette nouvelle région soviétique.

21 La lutte contre l'héritage de la culture germanique est devenue en quelque sorte une continuation de la propagande historique. On s'attaqua tout d'abord à la toponymie de l'ancienne Prusse-Orientale. Dès les années 1946-1947 eut lieu une campagne de changement des noms des localités, des rues, des places, des cours d'eau et autres noms géographiques. Cette campagne ne visait pas un anéantissement total, puisqu'une petite partie des noms anciens, pour l'essentiel des noms de cours d'eau, a été gardée, et certaines appellations nouvelles ont été créées en consonance avec les noms 
allemands (Domnau - Domnovo, Kastanienallee - Avenue des châtaigniers, etc.). Mais l'apparition de noms russes sur la carte de la région a reçu l'approbation sincère et unanime des colons. Les paroles suivantes, par exemple, étaient probablement sincères et non imposées d'en haut: "Nous saluons l'attribution d'un nom russe à la ville principale de la région, qui était habitée par les tribus slaves déjà dans l'Antiquité, cette ville dont le commandant était le père du grand chef militaire russe Suvorov ». À cet égard, on mentionnera ce commentaire d'I. Belintseva : "L'importance culturelle de cette démarche strictement pratique en apparence - le changement des noms inconnus, indistincts, muets pour la conscience culturelle de la nouvelle population est essentielle. On sait que le changement de nom, l'attribution d'une nouvelle désignation à un objet ou à une notion est un signe de son appropriation définitive ».

C'est dans le contexte de telles directives socio-psychologiques qu'il faut examiner la suppression des enseignes allemandes, des inscriptions en relief sur les façades des maisons et d'autres traces graphiques d'un passé étranger. Elle était considérée comme nécessaire pour la validation d'un nouveau système de signes propre aux Russes. Ainsi le secrétaire du comité du parti Tiourikov disait avec indignation à la conférence régionale sur le travail de propagande (du 15 janvier 1948) : «Quand vous arrivez à la gare du Sud, vous ne voyez pas que c'est Kaliningrad... Nous avons appelé l'ancienne ville de Königsberg Kaliningrad, mais on ne le voit nulle part. Vous allez peut-être me dire que c'est une question secondaire, mais des Russes arrivent ici et ils s'attendent à voir des appellations russes, qu'on ne voit pas chez nous ». Les traces de la sémiotique allemande allaient sauter aux yeux encore pendant de longues années : «Dans les rues de la ville on peut bien souvent voir des inscriptions, des enseignes, des bas-reliefs allemands. Il est grand temps d'enlever tout cela des rues de notre ville soviétique " (1952) ; « Notre région existe depuis déjà dix-huit ans, mais sur certains bâtiments dans les villes, les centres d'arrondissements, les localités, on trouve aujourd'hui encore des inscriptions allemandes. Au centre de la ville de Gousev tout le monde peut lire : "Mein Haus ist meine Welt", ce qui signifie: "Ma maison est ma forteresse"4. Pourquoi les komsomols de Gousev acceptent-ils ce slogan qui fait la propagande de l'idéologie des junkers prussiens? On trouve des inscriptions allemandes dans tous les districts. Il faut les effacer !» (1963). Ce problème n'a perdu son acuité que dans les années 1970.

Par ailleurs, la lutte contre l'héritage germanique se manifestait par la destruction de la structure des paysages, le démantèlement des œuvres de la sculpture monumentale idéologiquement étrangères à l'esprit soviétique, la liquidation des collections des musées, des fonds d'archives et des bibliothèques sous forme de donations ou de transmissions «temporaires » de ces fonds aux établissements culturels de Moscou, de Leningrad, de la Lituanie ou de la Pologne (voir aussi, à ce sujet, les articles de Garber et de Didier).

Le destin de l'architecture germanique mérite une attention particulière. Le côté artistique et technologique de ce problème a été étudié en détail, et de façon relativement objective, par I. Belintseva. Voici ce qu'elle en dit :

Conformément aux goûts esthétiques et aux besoins des nouveaux habitants [...] on modifiait la hauteur et la silhouette de certains bâtiments, et des locaux intérieurs étaient replanifiés. On installait dans les appartements des salles de bains et des cabines de douches séparées qui remplaçaient les salles de bains communes, qui se trouvaient chez les Allemands dans les sous-sols des maisons. On divisait les grands appartements en plusieurs petits. On rejetait de façon générale les appartements mansardés caractéristiques de la construction européenne. Leur organisation était 
officiellement considérée comme "malcommode et incompatible avec les normes". La liquidation des hauts toits de tuiles et des mansardes, caractéristiques de l'image architecturale des villes de la région baltique, changeait radicalement la silhouette globale du paysage municipal, la privant de sa typicité locale, et en conséquence des marques germaniques non désirées et étrangères aux yeux des nouveaux habitants russes.

À la fin des années 1940 et au début des années 1950 on a défini un nouvel alignement des façades, certains bâtiments ont été surélevés par des étages supplémentaires afin qu'ils soient plus représentatifs. Les bâtiments construits à Königsberg avant la guerre dans le style constructiviste ont reçu, conformément aux goûts des années 1950, des façades "enrichies" avec un système architectonique de composition tout à fait différent. À la place des plans muraux auparavant divisés par un vitrage horizontal modeste, sont apparues des compositions réunissant des détails architecturaux empruntés au classicisme, avec de nombreux balcons, des loggias, des corniches en bois ou sculptées. L'appréciation de la valeur des bâtiments reconstruits était en corrélation stricte avec les préférences esthétiques de l'époque d'après-guerre.

L'héritage national de l'architecture russe ancienne et celui de la période classique semblaient être les sources des nouvelles formes architecturales particulièrement dignes d'attention. Les styles inacceptables pour l'imitation ainsi que pour la restauration étaient ceux $\mathrm{du} 19^{\mathrm{e}}$ siècle, comme le néogothique, ainsi que le modernisme et le constructivisme des années 1920. On aspirait donc à reconstruire totalement les bâtiments néogothiques et constructivistes de l'ancienne Königsberg.

25 Ainsi, la relation à l'esthétique de l'héritage urbain de la Prusse-Orientale était ambiguë. Dans cette relation s'entremêlaient des éléments de destruction et de transformation. Néanmoins, les bâtiments et les ensembles architecturaux les plus proches de la tradition artistique des colons ou esthétiquement "neutres " ont été conservés sous leur aspect originel.

L'architecture religieuse, elle, a connu un sort un peu différent. D'après l'évaluation faite en 1945 par A.P. Bakhtine, employé des Archives d'État de la région de Kaliningrad, sur le territoire de la région se trouvaient 223 bâtiments de culte identifiés par lui en tant qu' "églises luthériennes » (les autres églises, les chapelles et les temples n'étaient donc pas pris en compte). Selon lui, après la fin des bombardements, 124 de ces églises luthériennes étaient restées «intactes ", 74 étaient endommagées et l'état des 24 églises restantes n'était pas connu. Malheureusement, l'auteur donne un chiffre " aveugle» (sans nommer les églises en question); c'est pourquoi on ne peut pas le vérifier. En guise de comparaison, on peut se référer à un fragment du certificat sur l'état du mouvement religieux écrit en mai 1948 par A. I. Glazkikh, le délégué du Conseil des affaires des cultes religieux auprès du Conseil des ministres de l'URSS : «Selon des données incomplètes, on compte à l'heure actuelle 116 temples de toutes les communautés religieuses dans la région de Kaliningrad, dont plus de 55 (c'est-à-dire $49 \%$ ) à Kaliningrad, et seulement 29 de ces temples sont utilisables ».

Jusqu'en 1950 la gestion des églises luthériennes allemandes faisait partie des compétences de ce même Glazkikh. Il connaissait bien la résolution de la présidence du Conseil suprême de la RSFSR du 16 décembre 1948, selon laquelle il était illégal d'utiliser les églises allemandes en tant que lieux de stockage, garages ou entreprises industrielles, mais qui permettait d'y héberger des établissements culturels. De plus, dans l'ordonnance du Conseil des affaires des cultes religieux du délégué de Kaliningrad (en février 1949), il était clairement indiqué que l'on pouvait affecter des bâtiments religieux non utilisés «uniquement à des institutions culturelles et civilisatrices 
comme les écoles, les musées, les bibliothèques, les fonds-dépôts de livres, les centres de conférences publiques, les archives, mais pas à des clubs et autres institutions semblables ». En outre, il fallait avoir l'autorisation du Conseil des affaires des cultes religieux pour la transmission ou le rééquipement des églises non utilisées.

Königsberg aujourd'hui : le village des pêcheurs et le pont jubilaire.

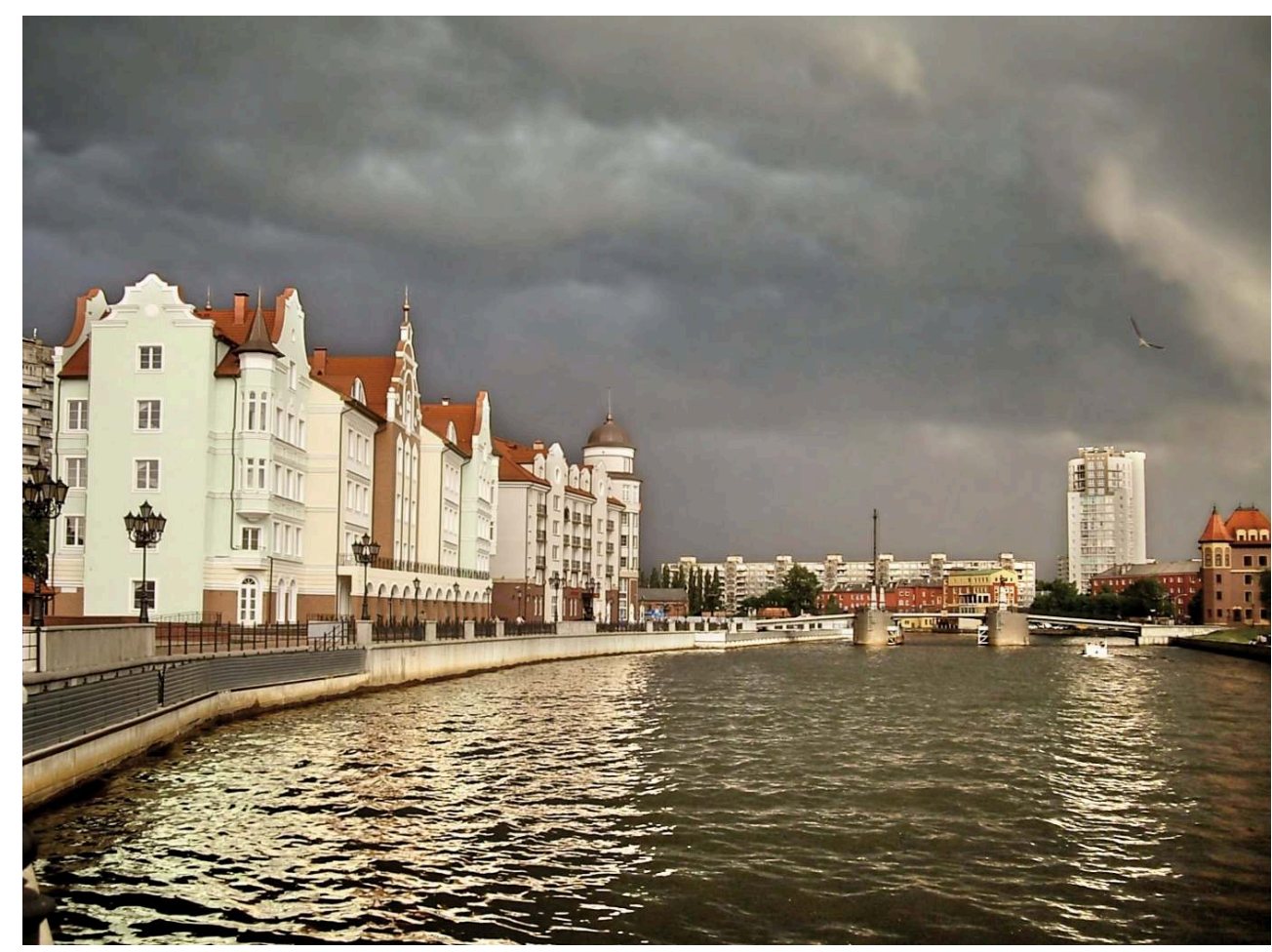

Ce pont piéton fut construit en 2005 pour le 750e anniversaire de la ville, sur les anciens piliers du pont Kaiserbrücke détruit pendant la guerre (architecte : Alexandre Bachine

Photographie Yuri Bardun

Cependant, après la déportation des Allemands, les catholiques et les luthériens soviétiques ne revendiquèrent pas instamment leurs droits sur les bâtiments de culte de la région de Kaliningrad, et les autorités ne donnèrent pas non plus les anciens temples aux croyants orthodoxes sous prétexte de leur appartenance à une autre confession. Dans le même temps, le nombre de bâtiments publics vacants disponibles se réduisait rapidement. Devant une telle situation, Glazkikh était obligé de sanctionner l'utilisation des églises allemandes par les diverses organisations d'État ou des collectivités locales, mais aussi par tous ceux à qui l'octroi des bâtiments religieux n'était pas recommandé. Ainsi, l'ancienne église luthérienne de Juditten, qui est devenue dès 1985 le premier temple orthodoxe de la région, avait changé de possesseur plusieurs fois à la fin des années 1940. D'abord entrepôt du bureau régional de tabac, elle devint ensuite un magasin de parfum, et ainsi de suite. La seule chose que Glazkikh faisait respecter, c'était l'interdiction de « la modification intérieure et extérieure » des bâtiments.

Après la liquidation de la fonction de délégué en 1950, le pouvoir absolu sur le destin des anciens lieux de culte allemands est passé aux mains des conseils locaux. Désormais il n'y avait plus personne pour veiller sur le maintien de leur aspect initial. Les églises et les chapelles qui abritaient des établissements culturels ont eu de la chance. 
Beaucoup de bâtiments religieux ont été délaissés. Petit à petit, ils ont commencé à tomber en ruines et à être démolis pour servir de matériaux de construction. D’après des témoins, la même église luthérienne de Juditten était déjà en ruines dans les années 1960 et a commencé alors à être utilisée par les habitants comme dépotoir. Son intégrité au moment de son transfert à l'Église orthodoxe ne dépassait pas 20 à $25 \%$ de l'état initial. En s'appuyant sur les souvenirs des anciens habitants de la région, A. Bakhtine est arrivé à la conclusion que sur 22 églises allemandes ayant survécu à la guerre, 7 étaient détruites vers 1950, à quoi s'ajoutaient «près de 26 églises luthériennes » dans les années 1960.

Reconnaissons pour être justes qu'il n'y avait pas de politique déterminée de destruction des lieux de culte allemands. Il faudrait plutôt parler de l'inaction des autorités qui n'ont pas entrepris les démarches nécessaires pour préserver les monuments d'architecture religieuse. Les gens simples ne protestaient pas. Une immigrante a dit à ce propos : «Le peuple russe ne les a pas préservés. Personne d'en haut n'avait donné l'instruction de "ne pas toucher". Et nous-mêmes ne pensions alors qu'à survivre. Je le regrette beaucoup aujourd'hui ». Au pays de l'athéisme officiel, une attitude différente à l'égard des églises n'était pas imaginable.

De plus, dans la région de Kaliningrad, les temples délaissés étaient ceux des étrangers, qui appartenaient autrefois à l'ennemi qui avait détruit les maisons et les villages des colons. De même que d'autres trophées culturels, les églises luthériennes allemandes ne provoquaient pas la vénération que certaines personnes éprouvaient encore à l'égard du sacré. Une femme qui faisait partie des premiers immigrants se rappelle, offensée :

Récemment il y a eu des articles écrits par des personnes qui n'avaient jamais vu ni cette région, ni cette terre, ni ces gens et qui disaient que les Russes n'avaient pas réussi à protéger la culture allemande. On nous accusait beaucoup, nous les premiers colons, de ne pas l'avoir préservée ou même de l'avoir détruite. Mais comment pouvait-on préserver cette culture? Premièrement, quand nous sommes arrivés dans notre coin - peut-être que c'était différent ailleurs - il y avait des combats qui ont duré quatre jours... On s'était installés dans une maison endommagée par un obus, avec un toit qui coulait, mais au moins les pièces étaient à sec... Mais la maison tombait en ruines peu à peu. En plus, nous sommes venues ici sans père, on était trois filles et une mère - que pouvait-on faire? Comment pouvions-nous restaurer cette culture allemande après la ruine, après la guerre? Moi, qui étais l'aînée, j'avais quinze ans à l'époque. Bien sûr, il y avait des cas de vandalisme, quand quelqu'un fouillait les cryptes et les tombes allemandes, je ne vous le cache pas. Mais pour l'essentiel, quels sentiments pouvait-on éprouver à l'égard des ennemis qui avaient tué nos pères au front, en nous laissant orphelins? Est-ce que nous pouvions respecter, vénérer et préserver la culture allemande? Non, nous ne le pouvions pas psychologiquement. [...]

Les deux parties, le pouvoir et le peuple, se contentaient tout à fait de la conception de l'histoire régionale qu'elles avaient réussi à créer. Le renforcement de l'élément étranger aurait pu augmenter la tension socio-psychologique dans la région. Il y avait aussi d'autres raisons pour que cette conception ne devienne pas plus «scientifique». L'historiographie soviétique, particulièrement au temps de Staline, était non seulement empreinte d'une forte idéologie, mais aussi d'un extrême utilitarisme [...] En 1958 un membre du komsomol disait: «Dans les cours qu'on nous envoie actuellement sur l'histoire de Kaliningrad, on trouve tous les rois prussiens, mais pas de sujets qui intéresseraient la jeunesse ». Dans le cas présent, on constate non tant l'ignorance de l'histoire prussienne orientale que le désir de ne pas considérer l'histoire comme une science utile en général. Les exemples en sont nombreux : ainsi, un autre membre du 
komsomol a déclaré lors d'une conférence régionale: «Dans ce manuel destiné aux enfants de 11-12 ans, il y a près de 400 dates et chiffres divers, plus de 115 noms de pharaons, de rois et de dieux, plus de 200 dénominations et définitions différentes. On étudie les lois d'Hammourabi, mais les enfants de cet âge ne connaissent même pas encore les lois soviétiques. On demande aux élèves de connaitre l'histoire des cinq mille boeufs d'Augias et du nettoyage de ses écuries, mais beaucoup d'entre eux n'ont aucune idée de ce que c'est que la ferme d'un kolkhoze moderne. On les oblige à connaître les ciseaux à main, les grattoirs et les pointes en silex dont les gens se servaient il y a 400000 ans, mais on n'apprend pas aux élèves à se servir du marteau-piqueur moderne, des scies électriques et d'autres outils qu'on utilise de nos jours, au $20^{\mathrm{e}}$ siècle ». Conception bien matérialiste...

Le pouvoir et le peuple étaient d'accord pour ce qui était du changement des noms et de la liquidation des inscriptions allemandes. En Pologne voisine et dans quelques autres pays la situation évoluait de manière analogue. Auparavant, les Allemands euxmêmes utilisaient ce moyen d'influence idéologique. Il suffit de rappeler un seul fait : au début de la Première Guerre mondiale, les villages russes de vieux croyants de la Prusse-Orientale ont reçu des noms allemands (Voynovo, par exemple, est devenu Eckertsdorf). Il n'y avait pas non plus de différends notables entre les simples Kaliningradois et les dirigeants politiques de la région au sujet de l'architecture et de la sculpture allemandes. Leur destruction partielle était perçue comme une action créatrice - une sorte de déblaiement de la terre pour les futurs champs labourés. Les colons de Kaliningrad avaient le droit moral de construire leur espace culturel sur les ruines, sans se retourner vers l'esprit et les traditions prussiennes. Cependant, les Russes ne pouvaient s'approprier ce nouvel espace qu'à la seule condition que l'idéologie soviétique réussît à l'intégrer à l'héritage national séculaire.

Pendant les années du stalinisme cette idéologie avait une certaine teinte de rhétorique nationaliste, permettait l'apparition des lueurs sporadiques de la mémoire historique et exploitait volontiers l'esthétique populaire, mais il n'y avait pas d'exploitation approfondie de la culture nationale ${ }^{5}$. Les autorités ne voulaient et ne pouvaient pas prendre en considération la foi des pères en tant que pivot spirituel des colons sur leurs nouvelles terres. C'est là que les intérêts et les possibilités du pouvoir étaient en dissonance avec les attentes du peuple ou au moins d'une partie de celui-ci. Un épisode en donne un bon exemple: en 1947, une rumeur (probablement correspondant à la réalité) s'était répandue parmi les habitants de la région, disant que sur le campanile à demi détruit d'une église catholique de Kaliningrad, il y avait une cloche emportée par les hitlériens du monastère de Kiev-Petchersk. Le délégué du Conseil des affaires des cultes religieux a commencé à recevoir des appels des militaires, des ingénieurs, des mécaniciens et d'autres colons qui proposaient leurs services pour faire descendre la cloche. Ils voyaient tous sans doute le trophée nazi comme un objet sacré d'une importance nationale, culturelle et religieuse. Sur cette question inhabituelle, Glazkikh a consulté la direction du Conseil des affaires des cultes religieux et de l'Eglise orthodoxe russe, et a reçu l'ordre de ne pas renvoyer la cloche à Kiev et de décider de son sort sur place. Par la suite, les fonctionnaires de Kaliningrad l'ont tout simplement oubliée. Personne n'a plus entendu parler de ce monument de la foi et de la culture russes, et ses traces sont aujourd'hui perdues. On aimerait croire qu'elles ne le sont pas définitivement. 


\section{NOTES}

1. Cet article est la reprise, légèrement réduite, de la version originale russe, jusqu'alors inédite en français, parue dans Prusse orientale et région de Kaliningrad au $X X^{e}$ siècle: recueil, in Études baltiques, $n^{\circ} 5$ (pages 56-69). Kaliningrad : Europe baltique, 2009.

2. République socialiste fédérative soviétique de Russie, État ancêtre de la Fédération de Russie au sein de l'URSS

3. En particulier, selon les données générales du service de migration du Comité exécutif régional pour les années 1946 1951, 3716 familles parmi celles qui furent envoyées dans la région pour travailler dans les institutions agricoles sont parties pendant cette période. 222 familles sont parties pour des raisons de santé, 168 ont été condamnées et expédiées par les organismes du ministère de la Sécurité de l'État, 30 sont revenues à cause de la convocation des chefs des familles dans l'armée soviétique et 3296 (près de $89 \%$ ) sont parties pour des raisons inconnues « sans autorisation ».

4. Ce qui, en l'occurrence, est une erreur (volontaire?) de traduction; il aurait fallu traduire par «Ma maison est mon univers» (NDT).

5. Dans l'Ouest saisi par la peur de «la menace russe», une telle politique était perçue comme celle de la «russification» de l'avant-poste occidental de l'URSS. Les presses européenne et américaine publiaient périodiquement des articles sur la destruction du patrimoine historique et culturel de l'ancienne Prusse-Orientale et l'implantation de la culture «barbare» russe. Le fragment suivant, tiré d'un article de Jour de France du 26 juillet 1959 et cité dans le feuilleton de I. Kouzmitchev Stéréotypes erronés à la mauvaise sauce (Kaliningradskaya Pravda, $\mathrm{n}^{\circ} 172$ [3180] du 30 août 1959), en est un exemple: "Depuis la fin de la guerre l'URSS essaie de "russifier" Königsberg qui est devenue à présent Kaliningrad. L'Union soviétique a installé là-bas des soldats russes déguisés en paysans, mais ils ont déserté à cause du manque de femmes. L'Union russe communiste de la jeunesse a été mise au courant, et après une large campagne propagandiste, cinq cents jeunes femmes de Minsk ont été envoyées à Kaliningrad. On les a accueillies dans les postes de l'office de l'état civil ». L'article était accompagné d'une photo représentant des jeunes filles marchant pendant le défilé sur la Place rouge. Un commentaire précisait qu'on leur avait confié la tâche du «soutien moral aux colons qui réalisaient la colonisation des nouvelles terres ». 